\title{
Exponential convexity of Petrović and related functional
}

\author{
Saad I Butt ${ }^{1 *}$, Josip Pečarić ${ }^{1,2}$ and Atiq Ur Rehman ${ }^{3}$
}

\section{* Correspondence:}

saadihsanbutt@gmail.com

${ }^{1}$ Abdus Salam School of

Mathematical Sciences, GC

University, Lahore, Pakistan

Full list of author information is

available at the end of the article

\begin{abstract}
We consider functionals due to the difference in Petrović and related inequalities and prove the log-convexity and exponential convexity of these functionals by using different families of functions. We construct positive semi-definite matrices generated by these functionals and give some related results. At the end, we give some examples.
\end{abstract}

Keywords: convex functions, divided difference, exponentially convex, functionals, log-convex functions, positive semi-definite

\section{Introduction}

First time exponentially convex functions are introduced by Bernstein [1]. Independently of Bernstein, but some what later Widder [2] introduced these functions, as a sub-class of convex functions in a given interval $(a, b)$, and denoted this class by $W_{a, b}$. After the initial development, there is a big gap in time before applications and examples of interest were constructed. One of the reasons is that, aside from absolutely monotone functions and completely monotone functions, as special classes of exponentially convex functions, there is no operative criteria to recognize exponential convexity of functions.

Definition 1. [[3], p. 373] A function $f:(a, b) \rightarrow \mathbb{R}$ is exponentially convex if it is continuous and

$$
\sum_{i, j=1}^{n} \xi_{i} \xi_{j} f\left(x_{i}+x_{j}\right) \geq 0
$$

for all $n \in \mathbb{N}$ and all choices $\xi_{i} \in \mathbb{R}$ and $x_{i}+x_{j} \in(a, b), 1 \leq i, j \leq n$.

Proposition 1.1. Let $f:(a, b) \rightarrow \mathbb{R}$. The following propositions are equivalent.

(i) $f$ is exponentially convex.

(ii) $f$ is continuous and

$$
\sum_{i, j=1}^{n} \xi_{i} \xi_{j} f\left(\frac{x_{i}+x_{j}}{2}\right) \geq 0
$$

for every $\xi_{i} \in \mathbb{R}$ and every $x_{i} \in(a, b), 1 \leq i \leq n$.

\section{Springer}

(C) 2011 Butt et al; licensee Springer. This is an Open Access article distributed under the terms of the Creative Commons Attribution License (http://creativecommons.org/licenses/by/2.0), which permits unrestricted use, distribution, and reproduction in any medium, provided the original work is properly cited. 
Proposition 1.2. If $f$ is exponentially convex, then the matrix

$$
\left[f\left(\frac{x_{i}+x_{j}}{2}\right)\right]_{i, j=1}^{n}
$$

is positive semi-definite. In particular,

$$
\operatorname{det}\left[f\left(\frac{x_{i}+x_{j}}{2}\right)\right]_{i, j=1}^{n} \geq 0
$$

for every $n \in \mathbb{N}, x_{i} \in(a, b), i=1, \ldots, n$.

Proposition 1.3. If $f:(a, b) \rightarrow(0, \infty)$ is an exponentially convex function, then $f$ is log-convex which means that for every $x, y \in(a, b)$ and all $\lambda \in(0,1)$

$$
f(\lambda x+(1-\lambda) y) \leq f(x)^{\lambda} f(y)^{1-\lambda} .
$$

We consider functionals due to the differences in the Petrović and related inequalities. These inequalities are given in the following theorems [[4], pp. 152-159].

Theorem 1.4. Let $I=(0, a] \subseteq \mathbb{R}$ be an interval, $\left(x_{1}, \ldots, x_{n}\right) \in I^{n}$, and $\left(p_{1}, \ldots, p_{n}\right)$ be a non-negative $n$-tuple such that

$$
\sum_{i=1}^{n} p_{i} x_{i} \in I \quad \text { and } \quad \sum_{i=1}^{n} p_{i} x_{i} \geq x_{j} \text { for } j=1, \ldots, n .
$$

If $f: I \rightarrow \mathbb{R}$ be a function such that $f(x) / x$ is an increasing for $x \in I$, then

$$
f\left(\sum_{i=1}^{n} p_{i} x_{i}\right) \geq \sum_{i=1}^{n} p_{i} f\left(x_{i}\right)
$$

Remark 1.5. Let us note that if $f(x) / x$ is a strictly increasing function for $x \in I$, then equality in (3) is valid if we have equalities in (2) instead of the inequalities, that is, $x_{1}$ $=\ldots=x_{n}$ and $\sum_{i=1}^{n} p_{i}=1$.

Theorem 1.6. Let $I=(0, a] \subseteq \mathbb{R}$ be an interval, $\left(x_{1}, \ldots, x_{n}\right) \in I^{n}$, such that $0<x_{1} \leq \ldots$ $\leq x_{n},\left(p_{1}, \ldots, p_{n}\right)$ be a non-negative $n$-tuple and $f: I \rightarrow \mathbb{R}$ be a function such that $f(x) / x$ is an increasing for $x \in I$.

(i) If there exists an $m(\leq n)$ such that

$$
0 \leq \bar{P}_{1} \leq \bar{P}_{2} \leq \cdots \leq \bar{P}_{m} \leq 1, \quad \bar{P}_{m+1}=\cdots=\bar{P}_{n}=0,
$$

where $P_{k}=\sum_{i=1}^{k} p_{i}, \bar{P}_{k}=P_{n}-P_{k-1}(k=2, \ldots, n)$ and $\bar{P}_{1}=P_{n}$, then (3) holds.

(ii) If there exists an $m(\leq n)$ such that

$$
\bar{P}_{1} \geq \bar{P}_{2} \geq \cdots \geq \bar{P}_{m} \geq 1, \quad \bar{P}_{m+1}=\cdots=\bar{P}_{n}=0,
$$

then the reverse of inequality in (3) holds.

Theorem 1.7. Let $I=(0, a] \subseteq \mathbb{R}$ be an interval, $\left(x_{1}, \ldots, x_{n}\right) \in I^{n}$, and $x_{1}-x_{2}-\ldots-x_{n}$ $\in I$. Also let $f: I \rightarrow \mathbb{R}$ be a function such that $f(x) / x$ is an increasing for $x \in I$. Then 


$$
f\left(x_{1}-\sum_{i=2}^{n} x_{i}\right) \leq f\left(x_{1}\right)-\sum_{i=2}^{n} f\left(x_{i}\right)
$$

Remark 1.8. If $f(x) / x$ is a strictly increasing function for $x \in I$, then strict inequality holds in (6).

Theorem 1.9. Let $I=(0, a] \subseteq \mathbb{R}$ be an interval, $\left(x_{1}, \ldots, x_{n}\right) \in I^{n},\left(p_{1}, \ldots, p_{n}\right)$ and $\left(q_{1}\right.$, ..., $q_{n}$ ) be non-negative $n$-tuples such that (2) holds. If $f: I \rightarrow \mathbb{R}$ be an increasing function, then

$$
\sum_{i=1}^{n} q_{i} f\left(\sum_{i=1}^{n} p_{i} x_{i}\right) \geq \sum_{i=1}^{n} q_{i} f\left(x_{i}\right)
$$

Remark 1.10. If $f$ is a strictly increasing function on I and all $x_{i}$ 's are not equal, then we obtain strict inequality in (7).

Theorem 1.11. Let $I=[0, a] \subseteq \mathbb{R}$ be an interval, $\left(x_{1}, \ldots, x_{n}\right) \in I^{n}$, and $\left(p_{1}, \ldots, p_{n}\right)$ be a non-negative $n$-tuple such that (2) holds.

If $f$ is a convex function on $I$, then

$$
f\left(\sum_{i=1}^{n} p_{i} x_{i}\right) \geq \sum_{i=1}^{n} p_{i} f\left(x_{i}\right)+\left(1-\sum_{i=1}^{n} p_{i}\right) f(0) .
$$

Remark 1.12. In the above theorem, if $f$ is a strictly convex, then inequality in (8) is strict, if all $x_{i}$ 's are not equal or $\sum_{i=1}^{n} p_{i} \neq 1$.

Theorem 1.13. Let $I \subseteq \mathbb{R}$ be an interval, $0 \in I$, $f$ be a convex function on $I, h:[a . b]$ $\rightarrow I$ be continuous and monotonic with $h\left(t_{0}\right)=0, t_{0} \in[a, b]$ be fixed, $g$ be a function of bounded variation and

$$
G(t):=\int_{a}^{t} \mathrm{~d} g(x), \quad \bar{G}(t):=\int_{t}^{b} \mathrm{~d} g(x) .
$$

(a) If $\int_{a}^{b} h(t) \mathrm{d} g(t) \in$ Iand

$$
0 \leq G(t) \leq 1 \quad \text { for } \quad a \leq t \leq t_{0}, \quad 0 \leq \bar{G}(t) \leq 1 \quad \text { for } \quad t_{0}<t \leq b,
$$

then we have

$$
\int_{a}^{b} f(h(t)) \mathrm{d} g(t) \geq f\left(\int_{a}^{b} h(t) \mathrm{d} g(t)\right)+\left(\int_{a}^{b} \mathrm{~d} g(t)-1\right) f(0) .
$$

(b) If $\int_{a}^{b} h(t) \mathrm{d} g(t) \in$ Iand either 
there exists an $s \leq t_{0}$ such that $G(t) \leq 0$ for $t<s$,

$$
G(t) \geq 1 \quad \text { for } \quad s \leq t \leq t_{0} \text { and } \bar{G}(t) \leq 0 \text { for } t>t_{0}
$$

$$
\begin{aligned}
& \text { or } \\
& \text { there exists an } s \geq t_{0} \text { such that } G(t) \leq 0 \text { for } t<t_{0}, \\
& \qquad \bar{G}(t) \geq 1 \text { for } t_{0}<t<s \text {, and } \bar{G}(t) \leq 0 \text { for } t \geq s,
\end{aligned}
$$

then the reverse of the inequality in (10) holds.

In this paper, we consider certain families of functions to prove log-convexity and exponential convexity of functionals due to the differences in inequalities given in Theorems 1.4-1.13. We construct positive semi-definite matrices generated by these functionals. Also by using log-convexity of these functionals, we prove monotonicity of the expressions introduced by these functionals. At the end, we give some examples.

\section{Main results}

Let $I \subseteq \mathbb{R}$ be an interval and $f: I \rightarrow \mathbb{R}$ be a function. Then for distinct points $u_{i} \in I, i$ $=0,1,2$, the divided differences in first and second order are defined as follows:

$$
\begin{gathered}
{\left[u_{i}, u_{i+1}, f\right]=\frac{f\left(u_{i+1}\right)-f\left(u_{i}\right)}{u_{i+1}-u_{i}} \quad(i=0,1),} \\
{\left[u_{0}, u_{1}, u_{2}, f\right]=\frac{\left[u_{1}, u_{2}, f\right]-\left[u_{0}, u_{1}, f\right]}{u_{2}-u_{0}} .}
\end{gathered}
$$

The values of the divided differences are independent of the order of the points $u_{0}$, $u_{1}, u_{2}$ and may be extended to include the cases when some or all points are equal, that is

$$
\left[u_{0}, u_{0}, f\right]=\lim _{u_{1} \rightarrow u_{0}}\left[u_{0}, u_{1}, f\right]=f^{\prime}\left(u_{0}\right),
$$

provided that $f$ exists.

Now passing through the limit $u_{1} \rightarrow u_{0}$ and replacing $u_{2}$ by $u$ in (13), we have [[4], p. 16]

$$
\left[u_{0}, u_{0}, u, f\right]=\lim _{u_{1} \rightarrow u_{0}}\left[u_{0}, u_{1}, u, f\right]=\frac{f(u)-f\left(u_{0}\right)-\left(u-u_{0}\right) f^{\prime}\left(u_{0}\right)}{\left(u-u_{0}\right)^{2}}, \quad u \neq u_{0},
$$

provided that $f$ exists. Also passing to the limit $u_{i} \rightarrow u(i=0,1,2)$ in (13), we have

$$
[u, u, u, f]=\lim _{u_{i} \rightarrow u}\left[u_{0}, u_{1}, u_{2}, f\right]=\frac{f^{\prime \prime}(u)}{2},
$$

provided that $f^{\prime \prime}$ exists.

One can note that if for all $u_{0}, u_{1} \in I,\left[u_{0}, u_{1}, f\right] \geq 0$, then $f$ is increasing on $I$ and if for all $u_{0}, u_{1}, u_{2} \in I,\left[u_{0}, u_{1}, u_{2}, f\right] \geq 0$, then $f$ is convex on $I$.

$\left(M_{1}\right)$ Under the assumptions of Theorem 1.4, with all $x_{i}^{\prime}$ s not equal, we define a linear functional as 


$$
\mathcal{P}_{1}(f)=f\left(\sum_{i=1}^{n} p_{i} x_{i}\right)-\sum_{i=1}^{n} p_{i} f\left(x_{i}\right) .
$$

$\left(M_{2}\right)$ Under the assumptions of Theorem 1.6, with all $x_{i}^{\prime}$ 's not equal and (4) is valid, we define a linear functional as

$$
\mathcal{P}_{2}(f)=\mathcal{P}_{1}(f) .
$$

$\left(M_{3}\right)$ Under the assumptions of Theorem 1.6, with all $x_{i}^{\prime}$ s not equal and (5) is valid, we define a linear functional as

$$
\mathcal{P}_{3}(f)=-\mathcal{P}_{1}(f) .
$$

$\left(M_{4}\right)$ Under the assumptions of Theorem 1.7, with all $x_{i}^{\prime}$ 's not equal, we define a linear functional as

$$
\mathcal{P}_{4}(f)=f\left(x_{1}\right)-\sum_{i=2}^{n} f\left(x_{i}\right)-f\left(x_{1}-\sum_{i=2}^{n} x_{i}\right) .
$$

$\left(M_{5}\right)$ Under the assumptions of Theorem 1.9, with all $x_{i}^{\prime}$ 's not equal, we define a linear functional as

$$
\mathcal{P}_{5}(f)=\sum_{i=1}^{n} q_{i} f\left(\sum_{i=1}^{n} p_{i} x_{i}\right)-\sum_{i=1}^{n} q_{i} f\left(x_{i}\right)
$$

$\left(M_{6}\right)$ Under the assumptions of Theorem 1.11 , with all $x_{i}^{\prime}$ s not equal, we define a linear functional as

$$
\mathcal{P}_{6}(f)=f\left(\sum_{i=1}^{n} p_{i} x_{i}\right)-\sum_{i=1}^{n} p_{i} f\left(x_{i}\right)-\left(1-\sum_{i=1}^{n} p_{i}\right) f(0) .
$$

$\left(M_{7}\right)$ Under the assumptions of Theorem 1.13, such that (9) is valid, we define a linear functional as

$$
\mathcal{P}_{7}(f)=\int_{a}^{b} f(h(t)) \mathrm{d} g(t)-f\left(\int_{a}^{b} h(t) \mathrm{d} g(t)\right)-\left(\int_{a}^{b} \mathrm{~d} g(t)-1\right) f(0) .
$$

$\left(M_{8}\right)$ Under the assumptions of Theorem 1.13, such that (11) or (12) is valid, we define a linear functional as

$$
\mathcal{P}_{8}(f)=-\mathcal{P}_{7}(f) .
$$

Remark 2.1. Under the assumptions of $\left(M_{k}\right)$ for $k=1,2,3,4$, if $f(u) / u$ is an increasing function for $u \in I$, then

$$
\mathcal{P}_{k}(f) \geq 0, \text { for } k=1,2,3,4 \text {. }
$$

If $f(u) / u$ is strictly increasing for $u \in I$ and all $x_{i}$ 's are not equal or $\sum_{1}^{n} p_{i} \neq 1$ then strict inequality holds in the above expression.

Remark 2.2. Under the assumptions of $\left(M_{5}\right)$, if $f$ is an increasing function on $I$, then

$$
\mathcal{P}_{5}(f) \geq 0 .
$$


If $f$ is strictly increasing function on I and all $x_{i}$ 's are not equal, then we obtain strict inequality in the above expression.

Remark 2.3. Under the assumptions of $\left(M_{k}\right)$ for $k=6,7,8$, if $f$ is a convex function on $I$, then

$$
\mathcal{P}_{k}(f) \geq 0 \text { for } k=6,7,8 .
$$

If $f$ is strictly increasing function on I and all $x_{i}$ 's are not equal, then we obtain strict inequality in the above expression for $\mathcal{P}_{6}(f)$.

The following lemma is nothing more than the discriminant test for the non-negativity of second-order polynomials.

Lemma 2.4. Let $I \subseteq \mathbb{R}$ be an interval. A function $f: I \rightarrow(0, \infty)$ is log-convex in $J$-sense on I, that is, for each $r, t \in I$

$$
f(r) f(t) \geq f^{2}\left(\frac{t+r}{2}\right)
$$

if and only if, the relation

$$
m^{2} f(t)+2 m n f\left(\frac{t+r}{2}\right)+n^{2} f(r) \geq 0
$$

holds for each $m, n \in \mathbb{R}$ and $r, t \in I$.

To define different families of functions, let $I \subseteq \mathbb{R}$ and $(c, d) \subseteq \mathbb{R}$ be intervals. For distinct points $u_{0}, u_{1}, u_{2} \in I$ we suppose

$\mathbf{D}_{1}=\left\{f_{t}: I \rightarrow \mathbb{R} \mid t \in(c, d)\right.$ and $t \mapsto\left[u_{0}, u_{1}, F_{t}\right]$ is log-convex in J-sense, where $F_{t}(u)$ $\left.=f_{t}(u) / u\right\}$.

$\mathbf{D}_{2}=\left\{f_{t}: I \rightarrow \mathbb{R} \mid t \in(c, d)\right.$ and $t \mapsto\left[u_{0}, u_{0}, F_{t}\right]$ is log-convex in J-sense, where $F_{t}(u)$ $=f_{t}(u) / u$ and $F_{t}^{\prime}$ exists $\}$.

$\mathbf{D}_{3}=\left\{f_{t}: I \rightarrow \mathbb{R} \mid t \in(c, d)\right.$ and $t \mapsto\left[u_{0}, u_{1}, f_{t}\right]$ is log-convex in J-sense $\}$.

$\mathbf{D}_{4}=\left\{f_{t}: I \rightarrow \mathbb{R} \mid t \in(c, d)\right.$ and $t \mapsto\left[u_{0}, u_{0}, f_{t}\right]$ is log-convex in J-sense, where $f_{t}^{\prime}$ exists\}.

$\mathbf{D}_{5}=\left\{f_{t}: I \rightarrow \mathbb{R} \mid t \in(c, d)\right.$ and $t \mapsto\left[u_{0}, u_{1}, u_{2}, f_{t}\right]$ is log-convex in J-sense $\}$.

$\mathbf{D}_{6}=\left\{f_{t}: I \rightarrow \mathbb{R} \mid t \in(c, d)\right.$ and $t \mapsto\left[u_{0}, u_{0}, u_{2}, f_{t}\right]$ is log-convex in J-sense, where $f_{t}^{\prime}$ exists\}.

$\mathbf{D}_{7}=\left\{f_{t}: I \rightarrow \mathbb{R} \mid t \in(c, d)\right.$ and $t \mapsto\left[u_{0}, u_{0}, u_{0}, f_{t}\right]$ is log-convex in J-sense, where $f_{t}^{\prime \prime}$ exists\}.

In this theorem, we prove log-convexity in J-sense, log-convexity and related results of the functionals associated with their respective families of functions.

Theorem 2.5. Let $\mathcal{P}_{k}$ be the linear functionals defined in $\left(M_{k}\right)$, associate the functionals with $\mathbf{D}_{i}$ in such a way that, for $k=1,2,3,4, f_{t} \in \mathbf{D}_{i}, i=1,2$, for $k=5, f_{t} \in \mathbf{D}_{i}$, $i=3,4$ and for $k=6,7,8, f_{t} \in \mathbf{D}_{i}, i=5,6,7$. Also for $k=7,8$, assume that the linear functionals are positive. Then, the following statements are valid:

(a) The functions $t \mapsto \mathcal{P}_{k}\left(f_{t}\right)$ are log-convex in J-sense on $(c, d)$.

(b) If the functions $t \mapsto \mathcal{P}_{k}\left(f_{t}\right)$ are continuous on $(c, d)$, then the functions $t \mapsto \mathcal{P}_{k}\left(f_{t}\right)$ are log-convex on $(c, d)$.

(c) If the functions $t \mapsto \mathcal{P}_{k}\left(f_{t}\right)$ are derivable on $(c, d)$, then for $t, r, u, v \in(c, d)$ such that $t \leq u, r \leq v$, we have 


$$
\mathfrak{B}_{k, i}\left(t, r ; f_{t}\right) \leq \mathfrak{B}_{k, i}\left(u, v ; f_{t}\right)
$$

where

$$
\mathfrak{B}_{k, i}\left(t, r ; f_{t}\right)= \begin{cases}\left(\frac{\mathcal{P}_{k}\left(f_{t}\right)}{\mathcal{P}_{k}\left(f_{r}\right)}\right)^{\frac{1}{t-r}}, & t \neq r, \\ \exp \left(\frac{\frac{d}{d_{t}}\left(\mathcal{P}_{k}\left(f_{t}\right)\right)}{\mathcal{P}_{k}\left(f_{t}\right)}\right), & t=r .\end{cases}
$$

Proof. (a) First, we prove log-convexity in J-sense of $t \mapsto \mathcal{P}_{k}\left(f_{t}\right)$ for $k=1,2,3$, 4. For this, we consider the families of functions defined in $\mathbf{D}_{1}$ and $\mathbf{D}_{2}$.

Choose any $m, n \in \mathbb{R}$, and $t, r \in(c, d)$, we define the function

$$
h(u)=m^{2} f_{t}(u)+2 m n f_{\frac{t+r}{2}}(u)+n^{2} f_{r}(u) .
$$

This gives

$$
\left[u_{0}, u_{1}, H\right]=m^{2}\left[u_{0}, u_{1}, F_{t}\right]+2 m n\left[u_{0}, u_{1}, F_{\frac{t+r}{2}}\right]+n^{2}\left[u_{0}, u_{1}, F_{r}\right],
$$

where $H(u)=h(u) / u$ and $F_{t}(u)=f_{t}(u) / u$.

Since $t \mapsto\left[u_{0}, u_{1}, F_{t}\right]$ is log-convex in J-sense, by Lemma 2.4 the right-hand side of above expression is non-negative. This implies $h(u) / u$ is an increasing function for $u \in$ I.

Thus by Remark 2.1

$$
\mathcal{P}_{k}(h) \geq 0 \text { for } k=1,2,3,4,
$$

this implies

$$
m^{2} \mathcal{P}_{k}\left(f_{t}\right)+2 m n \mathcal{P}_{k}\left(\frac{f_{t+r}}{2}\right)+n^{2} \mathcal{P}_{k}\left(f_{r}\right) \geq 0 .
$$

Now $\left[u_{0}, u_{1}, F_{t}\right]>0$ as it is log-convex, this implies $f_{t}(u) / u$ is strictly increasing for all $u \in I$ and $t \in(c, d)$. Also all $x_{i}^{\prime}$ 's are not equal and therefore by Remark 2.1, $\mathcal{P}_{k}\left(f_{t}\right)$ are positive valued, and hence, by Lemma 2.4 , the inequality (16) implies log-convexity in J-sense of the functions $t \mapsto \mathcal{P}_{k}\left(f_{t}\right)$ for $k=1,2,3,4$.

Now we prove $\log$-convexity in J-sense of $t \mapsto \mathcal{P}_{5}\left(f_{t}\right)$. For this, we consider the families of functions defined in $\mathbf{D}_{3}$ and $\mathbf{D}_{4}$. Following the same steps as above and having $H(u)=h(u)$, we have the log-convexity in J-sense of $\mathcal{P}_{5}\left(f_{t}\right)$ by using Remark 2.2 and Lemma 2.4.

At last, we prove log-convexity in J-sense of $t \mapsto \mathcal{P}_{k}\left(f_{t}\right)$ for $k=6,7$, 8. For this, we consider the families of Functions defined in $\mathbf{D}_{i}$ for $i=5,6,7$.

Choose any $m, n \in \mathbb{R}$, and $t, r \in(c, d)$, we define the function

$$
h(u)=m^{2} f_{t}(u)+2 m n f_{\frac{t+r}{2}}(u)+n^{2} f_{r}(u) .
$$

This gives

$$
\left[u_{0}, u_{1}, u_{2}, h\right]=m^{2}\left[u_{0}, u_{1}, u_{2}, f_{t}\right]+2 m n\left[u_{0}, u_{1}, u_{2}, \frac{f_{t+r}}{2}\right]+n^{2}\left[u_{0}, u_{1}, u_{2}, f_{r}\right] .
$$


Since $t \mapsto\left[u_{0}, u_{1}, u_{2}, f_{t}\right]$ is log-convex in J-sense, by Lemma 2.4 the right-hand side of above expression is non-negative. This implies $h$ is a strictly convex function on $I$.

Thus by Remark 2.3

$$
\mathcal{P}_{k}(h) \geq 0 \text { for } k=6,7,8,
$$

this implies

$$
m^{2} \mathcal{P}_{k}\left(f_{t}\right)+2 m n \mathcal{P}_{k}\left(\frac{f_{t+r}}{2}\right)+n^{2} \mathcal{P}_{k}\left(f_{r}\right) \geq 0 .
$$

Since $\mathcal{P}_{k}\left(f_{t}\right)$ are positive valued, we have by Lemma 2.4 and inequality (17) the logconvexity in J-sense of the functions $t \mapsto \mathcal{P}_{k}\left(f_{t}\right)$ for $k=6,7,8$.

(b) If $t \mapsto \mathcal{P}_{k}\left(f_{t}\right)$ are additionally continuous for $k=1, \ldots, 8$ and $\mathbf{D}_{i}$ 's associated with them, then these are log-convex, since J-convex continuous functions are convex functions.

(c) Since the functions $\log \mathcal{P}_{k}\left(f_{t}\right)$ are convex for $k=1, \ldots, 8$, and $\mathbf{D}_{i}$ 's associated with them, therefore for $t \leq u, r \leq v, t \neq r, u \neq v$, we have [[4], p.2],

$$
\frac{\log \mathcal{P}_{k}\left(f_{t}\right)-\log \mathcal{P}_{k}\left(f_{r}\right)}{t-r} \leq \frac{\log \mathcal{P}_{k}\left(f_{u}\right)-\log \mathcal{P}_{k}\left(f_{v}\right)}{u-v},
$$

concluding

$$
\mathfrak{B}_{k, i}\left(t, r ; f_{t}\right) \leq \mathfrak{B}_{k, i}\left(u, v ; f_{t}\right)
$$

Now if $t=r \leq u$, we apply $\lim _{r \rightarrow t}$, concluding,

$$
\mathfrak{B}_{k, i}\left(t, t ; f_{t}\right) \leq \mathfrak{B}_{k, i}\left(u, v ; f_{t}\right) .
$$

Other possible cases are treated similarly.

In order to define different families of functions related to exponential convexity, let $I \subseteq \mathbb{R}$ and $(c, d) \subseteq \mathbb{R}$ be any intervals. For distinct points $u_{0}, u_{1}, u_{2} \in I$ we suppose

$\mathbf{E}_{1}=\left\{f_{t}: I \rightarrow \mathbb{R} \mid t \in(c, d)\right.$ and $t \mapsto\left[u_{0}, u_{1}, F_{t}\right]$ is exponentially convex, where $F_{t}(u)=$ $\left.f_{t}(u) / u\right\}$.

$\mathbf{E}_{2}=\left\{f_{t}: I \rightarrow \mathbb{R} \mid t \in(c, d)\right.$ and $t \mapsto\left[u_{0}, u_{0}, F_{t}\right]$ is exponentially convex, where $F_{t}(u)=$ $f_{t}(u) / u$ and $F_{t}^{\prime}$ exists\}.

$\mathbf{E}_{3}=\left\{f_{t}: I \rightarrow \mathbb{R} \mid t \in(c, d)\right.$ and $t \mapsto\left[u_{0}, u_{1}, f_{t}\right]$ is exponentially convex $\}$.

$\mathbf{E}_{4}=\left\{f_{t}: I \rightarrow \mathbb{R} \mid t \in(c, d)\right.$ and $t \mapsto\left[u_{0}, u_{0}, f_{t}\right]$ is exponentially convex, where $f_{t}^{\prime}$ exists\}.

$\mathbf{E}_{5}=\left\{f_{t}: I \rightarrow \mathbb{R} \mid t \in(c, d)\right.$ and $t \mapsto\left[u_{0}, u_{1}, u_{2}, f_{t}\right]$ is exponentially convex $\}$.

$\mathbf{E}_{6}=\left\{f_{t}: I \rightarrow \mathbb{R} \mid t \in(c, d)\right.$ and $t \mapsto\left[u_{0}, u_{0}, u_{2}, f_{t}\right]$ is exponentially convex, where $f_{t}^{\prime}$ exists\}.

$\mathbf{E}_{7}=\left\{f_{t}: I \rightarrow \mathbb{R} \mid t \in(c, d)\right.$ and $t \mapsto\left[u_{0}, u_{0}, u_{0}, f_{t}\right]$ is exponentially convex, where $f_{t}^{\prime \prime}$ exists\}.

In this theorem, we prove the exponential convexity of the functionals associated with their respective families of functions. Also we define positive semi-definite matrices for these functionals and give some related results.

Theorem 2.6. Let $\mathcal{P}_{k}$ be the linear functionals defined in $\left(M_{k}\right)$, associate the functionals with $\mathbf{E}_{i}$ in such a way that, for $k=1,2,3,4, f_{t} \in \mathbf{E}_{i}, i=1,2$, for $k=5, f_{t} \in \mathbf{E}_{i}, i$ $=3,4$ and for $k=6,7,8, f_{t} \in \mathbf{E}_{i}, i=5,6,7$. Then, the following statements are valid: 
(a) If $t \mapsto \mathcal{P}_{k}\left(f_{t}\right)$ are continuous on $(c, d)$, then the functions $t \mapsto \mathcal{P}_{k}\left(f_{t}\right)$, are exponentially convex on $(c, d)$.

(b) For every $q \in \mathbb{N}$ and $t_{1}, \ldots, t_{q} \in(c, d)$, the matrices

$$
\left[\mathcal{P}_{k}\left(\frac{f_{t_{1}+t_{m}}}{2}\right)\right]_{l, m=1}^{q}
$$

are positive semi-definite. In particular

$$
\operatorname{det}\left[\mathcal{P}_{k}\left(\frac{f_{t_{1}+t_{m}}}{2}\right]_{l, m=1}^{s} \geq 0 \text { for } s=1,2, \ldots, q .\right.
$$

(c) If $t \mapsto \mathcal{P}_{k}\left(f_{t}\right)$ are positive derivable on $(c, d)$, then for $t, r, u, v \in(c, d)$ such that $t$ $\leq u, r \leq v$, we have

$$
\mathfrak{C}_{k, i}\left(t, r ; f_{t}\right) \leq \mathfrak{C}_{k, i}\left(u, v ; f_{t}\right)
$$

where $\mathfrak{C}_{k, i}\left(t, r ; f_{t}\right)$ is defined similarly as in (15).

Proof. (a) First, we prove exponential convexity of $t \mapsto \mathcal{P}_{k}\left(f_{t}\right)$ for $k=1,2,3$, 4. For this, we consider the families of functions defined in $\mathbf{E}_{1}$ and $\mathbf{E}_{2}$.

For any $n \in \mathbb{N}, \xi_{i} \in \mathbb{R}$ and $t_{i} \in(c, d), i=1, \ldots, n$, we define

$$
h(u)=\sum_{i, j=1}^{n} \xi_{i} \xi_{j} f_{\frac{t_{i}+t_{j}}{2}}(u) .
$$

This gives

$$
\left[u_{0}, u_{1}, H\right]=\sum_{i, j=1}^{n} \xi_{i} \xi_{j}\left[u_{0}, u_{1}, F_{\frac{t_{i}+t_{j}}{2}}\right],
$$

where $H(u)=h(u) / u$ and $F_{t}(u)=f_{t}(u) / u$.

Since $t \mapsto\left[u_{0}, u_{1}, F_{t}\right]$ is exponentially convex, right-hand side of the above expression is non-negative, which implies $h(u) / u$ is an increasing function on $I$.

Thus by Remark 2.1, we have

$$
\mathcal{P}_{k}(h) \geq 0, \text { for } k=1,2,3,4,
$$

thus

$$
\sum_{i, j=1}^{n} \xi_{i} \xi_{j} \mathcal{P}_{k}\left(f_{\frac{t_{i}+t_{j}}{2}}\right) \geq 0 .
$$

Hence $t \mapsto \mathcal{P}_{k}\left(f_{t}\right)$ is exponentially convex for $k=1,2,3,4$.

Now we prove exponential convexity of $t \mapsto \mathcal{P}_{5}\left(f_{t}\right)$. For this, we consider the families of functions defined in $\mathbf{E}_{3}$ and $\mathbf{E}_{4}$. Following the same steps as above and having $H(u)$ $=h(u)$, we have the exponential convexity of the $\mathcal{P}_{5}\left(f_{t}\right)$ by using Remark 2.2.

At last, we prove exponential convexity of $t \mapsto \mathcal{P}_{k}\left(f_{t}\right)$ for $k=6,7$, 8. For this, we consider the families of functions defined in $\mathbf{E}_{i}$ for $i=5,6,7$. 
For any $n \in \mathbb{N}, \xi_{i} \in \mathbb{R}$ and $t_{i} \in(c, d), i=1, \ldots, n$, we define

$$
h(u)=\sum_{i, j=1}^{n} \xi_{i} \xi_{j} \frac{f_{i}+t_{j}}{2}(u)
$$

This gives

$$
\left[u_{0}, u_{1}, u_{2}, h\right]=\sum_{i, j=1}^{n} \xi_{i} \xi_{j}\left[u_{0}, u_{1}, u_{2}, \frac{f_{i}+t_{j}}{2}\right] .
$$

Since $t \mapsto\left[u_{0}, u_{1}, u_{2}, f_{t}\right]$ is exponentially convex therefore right-hand side of the above expression is non-negative, which implies $h(u)$ is a strictly convex function on $I$.

Thus by Remark 2.3, we have

$$
\mathcal{P}_{k}(h) \geq 0 \text { for } k=6,7,8,
$$

thus

$$
\sum_{i, j=1}^{n} \xi_{i} \xi_{j} \mathcal{P}_{k}\left(f_{\frac{t_{i}+t_{j}}{2}}\right) \geq 0 .
$$

Hence $t \mapsto \mathcal{P}_{k}\left(f_{t}\right)$ are exponentially convex for $k=6,7,8$.

(b) It follows by Proposition 1.2.

(c) Since $t \mapsto \mathcal{P}_{k}\left(f_{t}\right)$ are positive derivable for $k=1, \ldots, 8$ with $\mathbf{E}_{i}$ 's associated with them, we have our conclusion using part $(c)$ of the Theorem 2.5.

\section{Examples}

In this section, we will vary on choices of families of functions in order to construct different examples of log and exponentially convex functions and related results.

Example 1. Let $t \in \mathbb{R}$ and $\phi_{t}:(0, \infty) \rightarrow \mathbb{R}$ be the function defined as

$$
\varphi_{t}(u)= \begin{cases}\frac{u^{t}}{t-1}, & t \neq 1 \\ u \log u, & t=1 .\end{cases}
$$

Then $\phi_{t}(u) / u$ is strictly increasing on $(0, \infty)$ for each $t \in \mathbb{R}$. One can note that $t \mapsto$ $\left[u_{0}, u_{0}, \phi_{t}(u) / u\right]$ is log-convex for all $t \in \mathbb{R}$. If we choose $f_{t}=\phi_{t}$ in Theorem 2.5, we get $\log$-convexity of the functionals $\mathcal{P}_{k}\left(\varphi_{t}\right)$ for $k=1,2,3$, 4, which have been proved in $[5,6]$.

Since $\left.\phi_{t}(u) / u\right)^{\prime}=u^{t-2}=\mathrm{e}^{(t-2) \log u}$, the mapping $t \mapsto\left(\phi_{t}(u) / u\right)^{\prime}$ is exponentially convex [7]. If we choose $f_{t}=\phi_{t}$ in Theorem 2.6, we get results that have been proved in $[6,8]$. Also we get $\mathfrak{C}_{1,2}\left(t, r ; \varphi_{t}\right)=A_{t, r}^{1}(\mathbf{x} ; \mathbf{p})$ for $t, r \neq 1$. By making substitution $x_{i} \mapsto x_{i}^{s}, t$ $\mapsto t / s, r \mapsto r / s$ and $s \neq 0, t, r \neq s$, we get $\mathfrak{C}_{1,2}\left(t, r ; \varphi_{t}\right)=A_{t, r}^{s}(\mathbf{x} ; \mathbf{p})$ for $t, r \neq s$, where $A_{t, r}^{s}(\mathbf{x} ; \mathbf{p})$ is defined in [5].

Similarly, $\mathfrak{C}_{4,2}\left(t, r ; \varphi_{t}\right)=C_{t, r}^{1}(\mathbf{x})$ for $t, r \neq 1$, and by substitution used above $\mathfrak{C}_{4,2}\left(t, r ; \varphi_{t}\right)=C_{t, r}^{s}(\mathbf{x})$ for $t, r \neq s$, where $C_{t, r}^{s}(\mathbf{x})$ is defined in [6].

Example 2. Let $t \in \mathbb{R}$ and $\beta_{t}:(0, \infty) \rightarrow \mathbb{R}$ be the function defined as

$$
\beta_{t}(u)=\left\{\begin{array}{l}
\frac{u^{t}}{t}, \quad t \neq 0 \\
\log u, t=0
\end{array}\right.
$$


Then, $\beta_{t}$ is strictly increasing on $(0, \infty)$ for each $t \in \mathbb{R}$. One can note that $t \mapsto\left[u_{0}, u_{0}\right.$, $\left.\beta_{t}\right]$ is log-convex for all $t \in \mathbb{R}$. If we choose $f_{t}=\beta_{t}$ in Theorem 2.5, we get log-convexity of the functional $\mathcal{P}_{5}\left(\beta_{t}\right)$, which have been proved in [9].

Since $\beta_{t}^{\prime}(u)=u^{t-1}=\mathrm{e}^{(t-1) \log u}$, the mapping $t \mapsto \beta_{t}^{\prime}$ is exponentially convex [7]. If we choose $f_{t}=\beta_{t}$ in Theorem 2.6, we get results that have been proved in [9]. Also we get $\mathfrak{C}_{5,4}\left(t, r ; \beta_{t}\right)=H_{t, r}(\mathbf{x} ; \mathbf{p} ; \mathbf{q})$ for $t, r \neq 0$, where $H_{t, r}(\mathbf{x} ; \mathbf{p} ; \mathbf{q})$ is defined in [9].

Example 3. Let $t \in(0, \infty)$ and $\delta_{t}:[0, \infty) \rightarrow \mathbb{R}$ be the function defined as

$$
\delta_{t}(u)= \begin{cases}\frac{u^{t}}{t(t-1)}, & t \neq 1, \\ u \log u, & t=1,\end{cases}
$$

with a convention that $0 \log 0=0$. Then $\delta_{t}$ is convex on $[0, \infty)$ for each $t \in(0, \infty)$. One can note that $t \mapsto\left[u_{0}, u_{0}, u_{0}, \delta_{t}\right]$ is log-convex for all $t \in(0, \infty)$. If we choose $f_{t}=$ $\delta_{t}$ in Theorem 2.5, we get log-convexity of the functionals $\mathcal{P}_{k}\left(\delta_{t}\right)$ for $k=6,7,8$, which have been proved in [10].

Since $\delta_{t}^{\prime \prime}(u)=u^{t-2}=\mathrm{e}^{(t-2) \log u}$, the mapping $t \mapsto \delta_{t}^{\prime \prime}$ is exponentially convex [7]. If we choose $f_{t}=\delta_{t}$ in Theorem 2.6, we get results that have been proved in $[8,10]$. Also we get $\mathfrak{C}_{6,7}\left(t, r ; \delta_{t}\right)=B_{t, r}^{1}(\mathbf{x} ; \mathbf{p})$ for $t, r \neq 1$. By making substitution $x_{i} \mapsto x_{i}^{s}, t \mapsto t / s, r \mapsto r / s$ and $s \neq 0, t, r \neq s$, we get $\mathfrak{C}_{6,7}\left(t, r ; \delta_{t}\right)=B_{t, r}^{s}(\mathbf{x} ; \mathbf{p})$ for $t, r \neq s$, where $B_{t, r}^{s}(\mathbf{x} ; \mathbf{p})$ is defined in [10].

Similarly, $\mathfrak{C}_{7,7}\left(t, r ; \delta_{t}\right)=F_{t, r}^{1}(a, b, h, g)$ for $t, r \neq 1$ and by substitution used above $\mathfrak{C}_{7,7}\left(t, r ; \delta_{t}\right)=F_{t, r}^{s}(a, b, h, g)$ for $t, r \neq s$, where $F_{t, r}^{s}(a, b, h, g)$ is defined in [6].

Example 4. Let $t \in(0, \infty)$ and $\zeta_{t}:(0, \infty) \rightarrow \mathbb{R}$ be the function defined as

$$
\zeta_{t}(u)= \begin{cases}\frac{u t^{-u}}{-\log t}, & t \neq 1, \\ u^{2}, & t=1 .\end{cases}
$$

One can note that $t \mapsto\left[u_{0}, u_{0}, \zeta_{t}(u) / u\right]$ is log-convex for all $t \in(0, \infty)$. If we choose $f_{t}$ $=\zeta_{t}$ in Theorem 2.5, we get log-convexity of the functionals $\mathcal{P}_{k}\left(\zeta_{t}\right)$ for $k=1,2,3,4$.

Since $\left.\zeta_{t}(u) / u\right)^{\prime}=t^{-u}$, the mapping $t \mapsto\left(\zeta_{t}(u) / u\right)^{\prime}$ is exponentially convex [7]. If we choose $f_{t}=\zeta_{t}$ in Theorem 2.6, we get exponential convexity of the functionals $\mathcal{P}_{k}\left(\zeta_{t}\right)$ for $k=1,2,3,4$.

For $\mathcal{P}_{1}\left(f_{t}\right)$ using the function $\zeta_{t}$ in Theorem 2.6, $\mathfrak{c}_{1,2}\left(t, r ; \zeta_{t}\right)$ in this particular case looks like

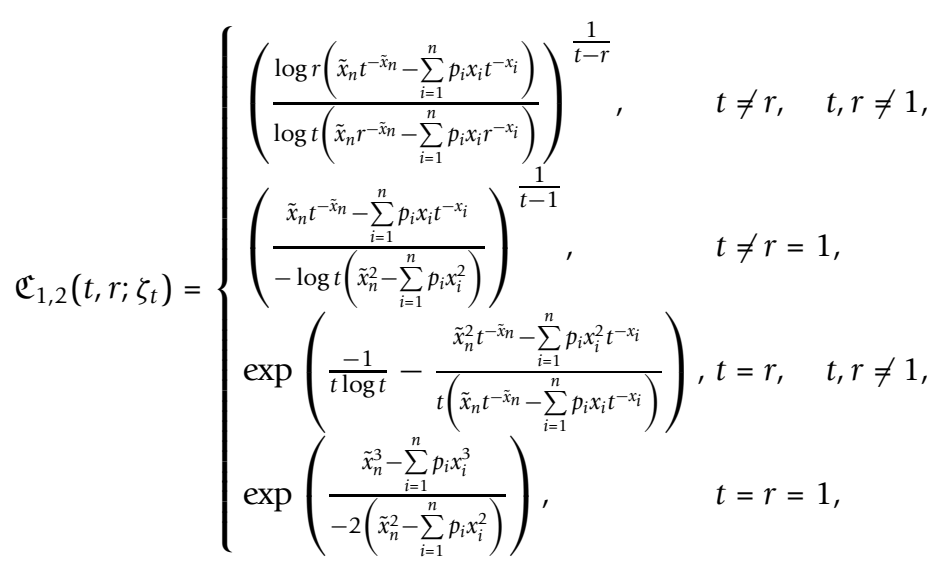

where $\tilde{x}_{n}=\sum_{i=1}^{n} p_{i} x_{i}$. 
For $\mathcal{P}_{4}\left(f_{t}\right)$ using the function $\zeta_{t}$ in Theorem 2.6, $\mathfrak{C}_{4,2}\left(t, r ; \zeta_{t}\right)$ in this particular case looks like

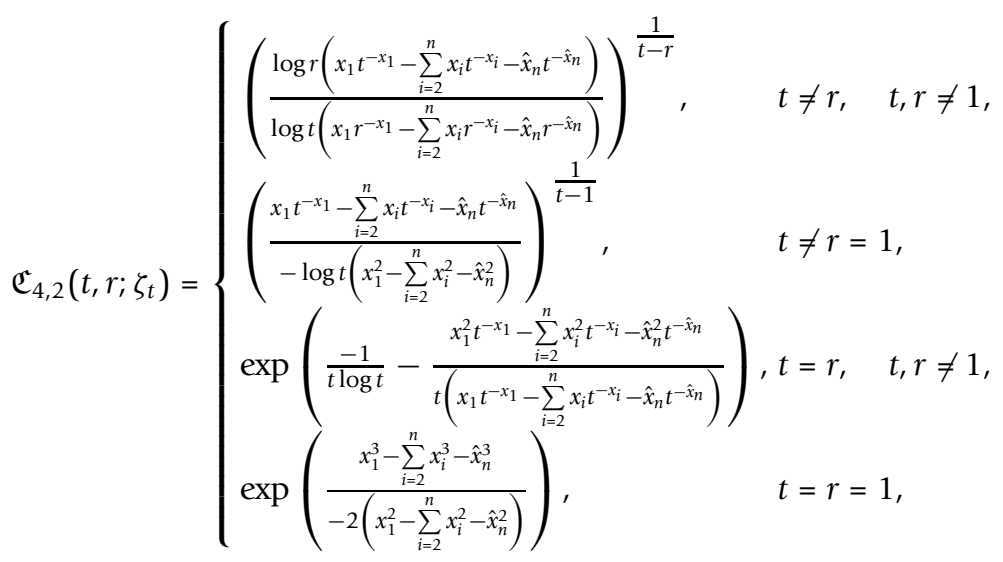

where $\hat{x}_{n}=\left(x_{1}-\sum_{i=2}^{n} x_{i}\right)$.

Example 5. Let $t \in(0, \infty)$ and $\theta_{t}:(0, \infty) \rightarrow \mathbb{R}$ be the function defined as

$$
\theta_{t}(u)= \begin{cases}\frac{t^{-u}}{-\log t}, & t \neq 1 \\ u, & t=1\end{cases}
$$

One can note that $t \mapsto\left[u_{0}, u_{0}, \theta_{t}\right]$ is log-convex for all $t \in(0, \infty)$, and if we choose $f_{t}$ $=\theta_{t}$ in Theorem 2.5, we get log-convexity of the functional $\mathcal{P}_{5}\left(\theta_{t}\right)$.

Since $\theta_{t}^{\prime}(u)=t^{-u}$, the mapping $t \mapsto \theta_{t}^{\prime}(u)$ is exponentially convex function [7]. If we choose $f_{t}=\theta_{t}$ in Theorem 2.6, we get exponential convexity of the functional $\mathcal{P}_{5}\left(\theta_{t}\right)$.

For $\mathcal{P}_{5}\left(f_{t}\right)$ using the function $\theta_{t}$ in Theorem 2.6, $\mathfrak{C}_{5,4}\left(t, r ; \theta_{t}\right)$ in this particular case looks like

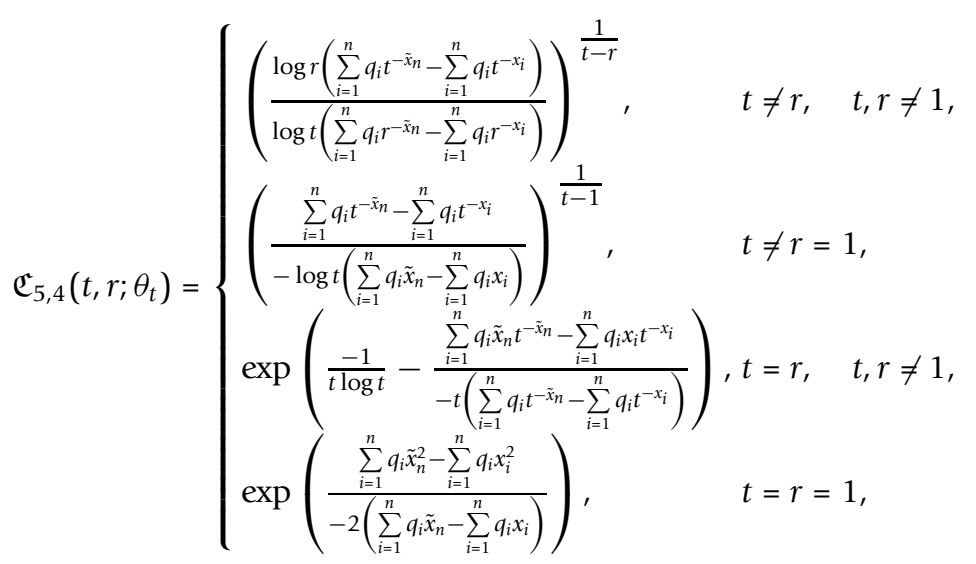

Where $\tilde{x}_{n}=\sum_{i=1}^{n} p_{i} x_{i}$.

Example 6. Let $t \in(0, \infty)$ and $\lambda_{t}:(0, \infty) \rightarrow \mathbb{R}$ be the function defined as

$$
\lambda_{t}(u)=\frac{u e^{-u \sqrt{t}}}{-\sqrt{t}}
$$

One can note that $t \mapsto\left[u_{0}, u_{0}, \lambda_{t}(u) / u\right]$ is log-convex for all $t \in(0, \infty)$. If we choose $f_{t}=\lambda_{t}$ in Theorem 2.5, we get log-convexity of the functionals $\mathcal{P}_{k}\left(\lambda_{t}\right)$ for $k=1,2,3,4$. 
Since $\left(\lambda_{t}(u) / u\right)^{\prime}=e^{-u \sqrt{t}}$, the mapping $t \mapsto\left(\lambda_{t}(u) / u\right)^{\prime}$ is exponentially convex function [7]. If we choose $f_{t}=\lambda_{t}$ in Theorem 2.6, we get exponential convexity of the functionals $\mathcal{P}_{k}\left(\lambda_{t}\right)$ for $k=1,2,3,4$.

For $\mathcal{P}_{1}\left(f_{t}\right)$ using the function $\lambda_{t}$ in Theorem 2.6, $\mathfrak{C}_{1,2}\left(t, r ; \lambda_{t}\right)$ in this particular case looks like

$$
\mathfrak{C}_{1,2}\left(t, r ; \lambda_{t}\right)=\left\{\begin{array}{l}
\left(\frac{\sqrt{r}\left(\tilde{x}_{n} \mathrm{e}^{-\tilde{x}_{n} \sqrt{t}}-\sum_{i=1}^{n} p_{i} x_{i} \mathrm{e}^{-x_{i} \sqrt{t}}\right)}{\sqrt{t}\left(\tilde{x}_{n} \mathrm{e}^{-\tilde{x}_{n} \sqrt{T}}-\sum_{i=1}^{n} p_{i} x_{i} \mathrm{e}^{-x_{i} \sqrt{T}}\right)}\right)^{\frac{1}{t-r}}, t \neq r, \\
\exp \left(\frac{-1}{2 t}-\frac{\tilde{x}_{n}^{2} \mathrm{e}^{-\tilde{x}_{n} \sqrt{t}}-\sum_{i=1}^{n} p_{i} x_{i}^{2} \mathrm{e}^{-x_{i} \sqrt{t}}}{2 \sqrt{t}\left(\tilde{x}_{n} \mathrm{e}^{-\tilde{x}_{n} \sqrt{t}}-\sum_{i=1}^{n} p_{i} x_{i} \mathrm{e}^{-x_{i} \sqrt{t}}\right)}\right), t=r,
\end{array}\right.
$$

Where $\tilde{x}_{n}=\sum_{i=1}^{n} p_{i} x_{i}$.

Now for $\mathcal{P}_{4}\left(f_{t}\right)$ using the function $\lambda_{t}$ in Theorem 2.6, $\mathfrak{C}_{4,2}\left(t, r ; \lambda_{t}\right)$ in this particular case looks like

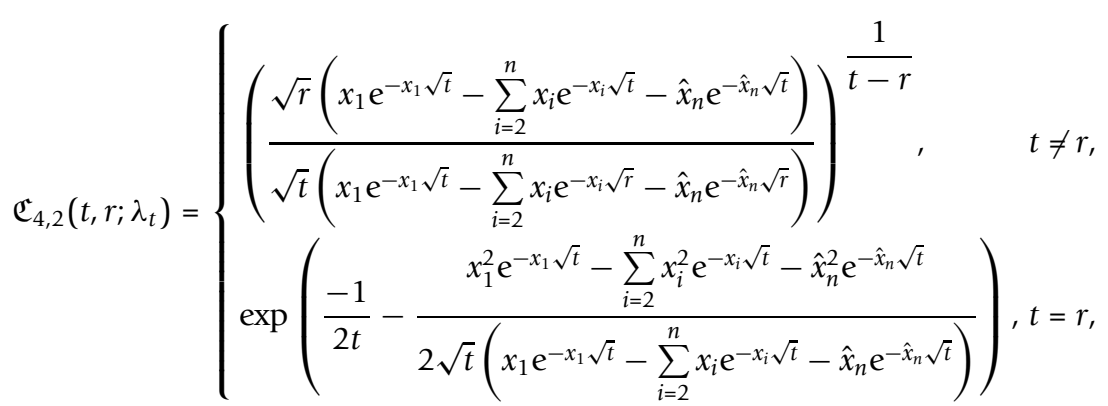

Where $\hat{x}_{n}=\left(x_{1}-\sum_{i=2}^{n} x_{i}\right)$.

Example 7. Let $t \in(0, \infty)$ and $\xi_{t}:(0, \infty) \rightarrow \mathbb{R}$, be the function defined as

$$
\xi_{t}(u)=\frac{e^{-u \sqrt{t}}}{-\sqrt{t}}
$$

One can note that $t \mapsto\left[u_{0}, u_{0}, \xi_{t}\right]$ is log-convex for all $t \in(0, \infty)$. If we choose $f_{t}=\xi_{t}$ in Theorem 2.5, we get log-convexity of the functional $\mathcal{P}_{5}\left(\xi_{t}\right)$.

Since $\xi_{t}^{\prime}(u)=\mathrm{e}^{-u \sqrt{t}}$, the mapping $t \mapsto \xi_{t}^{\prime}(u)$ is exponentially convex function [7]. If we choose $f_{t}=\xi_{t}$ in Theorem 2.6 we get exponential convexity of the functional $\mathcal{P}_{5}\left(\xi_{t}\right)$.

For $\mathcal{P}_{5}\left(f_{t}\right)$ using the function $\xi_{t}$ in Theorem 2.6, $\mathfrak{C}_{5,4}\left(t, r ; \xi_{t}\right)$ in this particular case looks like

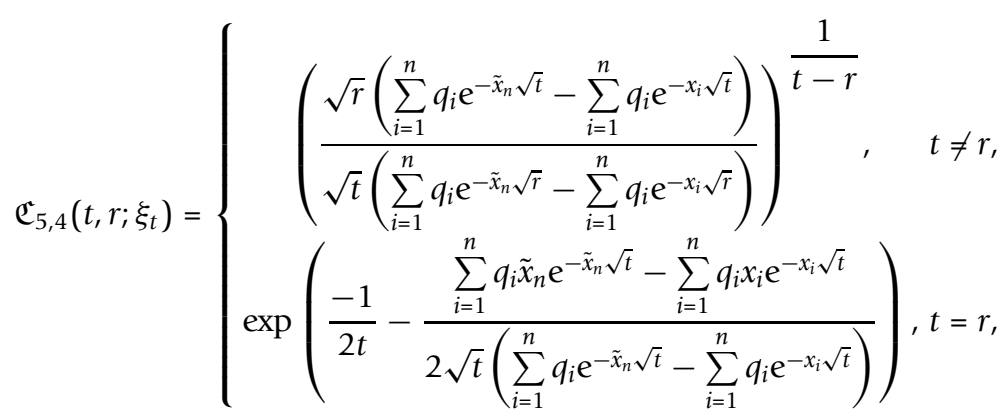

Where $\tilde{x}_{n}=\sum_{i=1}^{n} p_{i} x_{i}$. 
Example 8. Let $t \in \mathbb{R}$ and $\psi_{t}:(0, \infty) \rightarrow \mathbb{R}$ be the function defined as

$$
\psi_{t}(u)= \begin{cases}\frac{u e^{u t}}{t}, & t \neq 0 \\ u^{2}, & t=0\end{cases}
$$

One can note that $t \mapsto\left[u_{0}, u_{0}, \psi_{t}(u) / u\right]$ is log-convex for all $t \in \mathbb{R}$. If we choose $f_{t}=$ $\psi_{t}$ in Theorem2.5, we get $\log$-convexity of the functionals $\mathcal{P}_{k}\left(\psi_{t}\right)$ for $k=1,2,3,4$.

Since $\left(\psi_{t}(u) / u\right)^{\prime}=\mathrm{e}^{u t}$, the mapping $t \mapsto\left(\psi_{t}(u) / u\right)^{\prime}$ is exponentially convex function [7]. If we choose $f_{t}=\psi_{t}$ in Theorem 2.6 we get exponential convexity of the functionals $\mathcal{P}_{k}\left(\psi_{t}\right)$ for $k=1,2,3,4$.

For $\mathcal{P}_{1}\left(f_{t}\right)$ using the function $\psi_{t}$ in Theorem 2.6, $\mathfrak{C}_{1,2}\left(t, r ; \psi_{t}\right)$ in this particular case looks like

$$
\mathfrak{C}_{1,2}\left(t, r ; \psi_{t}\right)= \begin{cases}\left(\frac{r\left(\tilde{x}_{n} \mathrm{e}^{\tilde{n}_{n} t}-\sum_{i=1}^{n} p_{i} x_{i} \mathrm{e}^{x_{i} t}\right)}{t\left(\tilde{x}_{n} \mathrm{e}^{\tilde{e}_{n} r}-\sum_{i=1}^{n} p_{i} x_{i} \mathrm{e}^{x_{i} r}\right)}\right)^{\frac{1}{t-r}}, t \neq r, \quad t, r \neq 0, \\ \frac{\tilde{x}_{n} \tilde{x}^{\tilde{x}_{n} t}-\sum_{i=1}^{n} p_{i} x_{i} \mathrm{e}^{x_{i} t}}{t\left(\tilde{x}_{n}^{2}-\sum_{i=1}^{n} p_{i} x_{i}^{2}\right)}, \frac{1}{t-1}, & t \neq r=0, \\ \exp \left(\frac{-1}{t}+\frac{\tilde{x}_{n}^{2} \mathrm{e}^{\tilde{x}_{n} t}-\sum_{i=1}^{n} p_{i} x_{i}^{2} \mathrm{e}^{x_{i} t}}{\tilde{x}_{n} \mathrm{e}^{\tilde{x}_{n} t}-\sum_{i=1}^{n} p_{i} x_{i} \mathrm{e}^{x_{i} t}}\right), t=r, \quad t, r \neq 0, \\ \exp \left(\frac{\tilde{x}_{n}^{3}-\sum_{i=1}^{n} p_{i} x_{i}^{3}}{2\left(\tilde{x}_{n}^{2}-\sum_{i=1}^{n} p_{i} x_{i}^{2}\right)}\right), & t=r=0,\end{cases}
$$

Where $\tilde{x}_{n}=\sum_{i=1}^{n} p_{i} x_{i}$.

Now for $\mathcal{P}_{4}\left(f_{t}\right)$ using the function $\psi_{t}$ in Theorem 2.6, $\mathfrak{C}_{4,2}\left(t, r ; \psi_{t}\right)$ in this particular case looks like

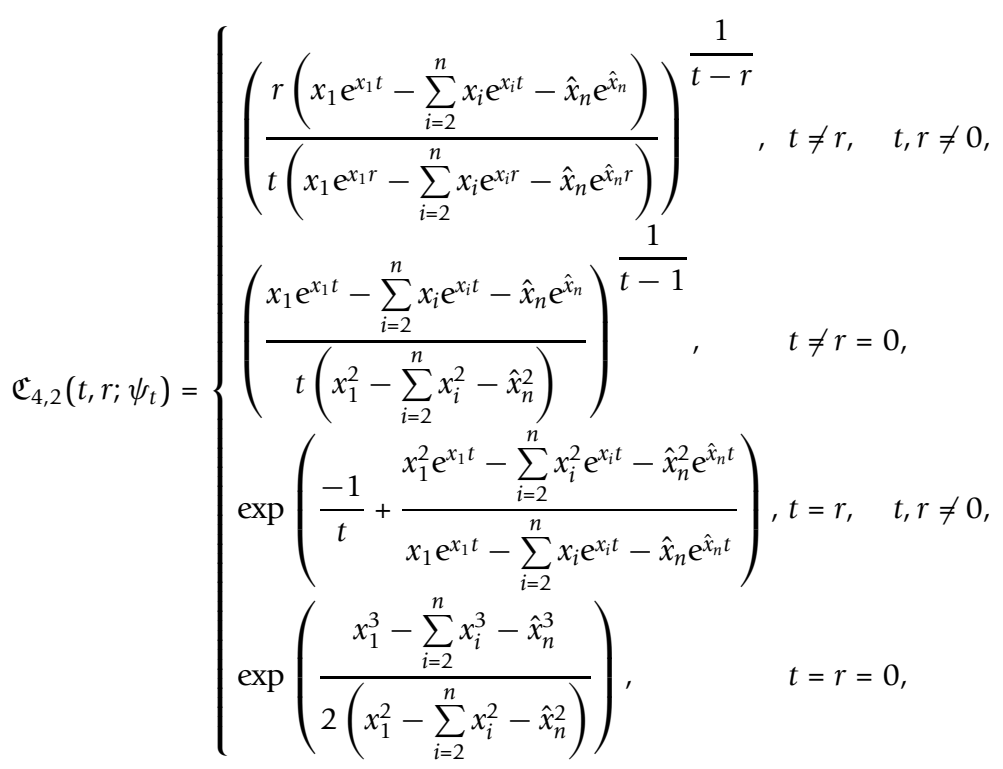


where $\hat{x}_{n}=\left(x_{1}-\sum_{i=2}^{n} x_{i}\right)$.

Example 9. Let $t \in \mathbb{R}$ and $\omega_{t}:(0, \infty) \rightarrow \mathbb{R}$ be the function defined as

$$
\omega_{t}(u)=\left\{\begin{array}{l}
\frac{\mathrm{e}^{u t}}{t}, t \neq 0 \\
u, t=0
\end{array}\right.
$$

One can note that $t \mapsto\left[u_{0}, u_{0}, \omega_{t}\right]$ is log-convex for all $t \in \mathbb{R}$. If we choose $f_{t}=\omega_{t}$ in Theorem 2.5, we get log-convexity of the functional $\mathcal{P}_{5}\left(\omega_{t}\right)$.

Since $\omega_{t}^{\prime}(u)=\mathrm{e}^{u t}$, the mapping $t \mapsto \omega_{t}^{\prime}(u)$ is exponentially convex function [7]. If we choose $f_{t}=\omega_{t}$ in Theorem 2.6 we get exponential convexity of the functional $\mathcal{P}_{5}\left(\omega_{t}\right)$.

For $\mathcal{P}_{5}\left(f_{t}\right)$ using the function $\omega_{t}$ in Theorem 2.6, $\mathfrak{C}_{5,4}\left(t, r ; \omega_{t}\right)$ in this particular case looks like

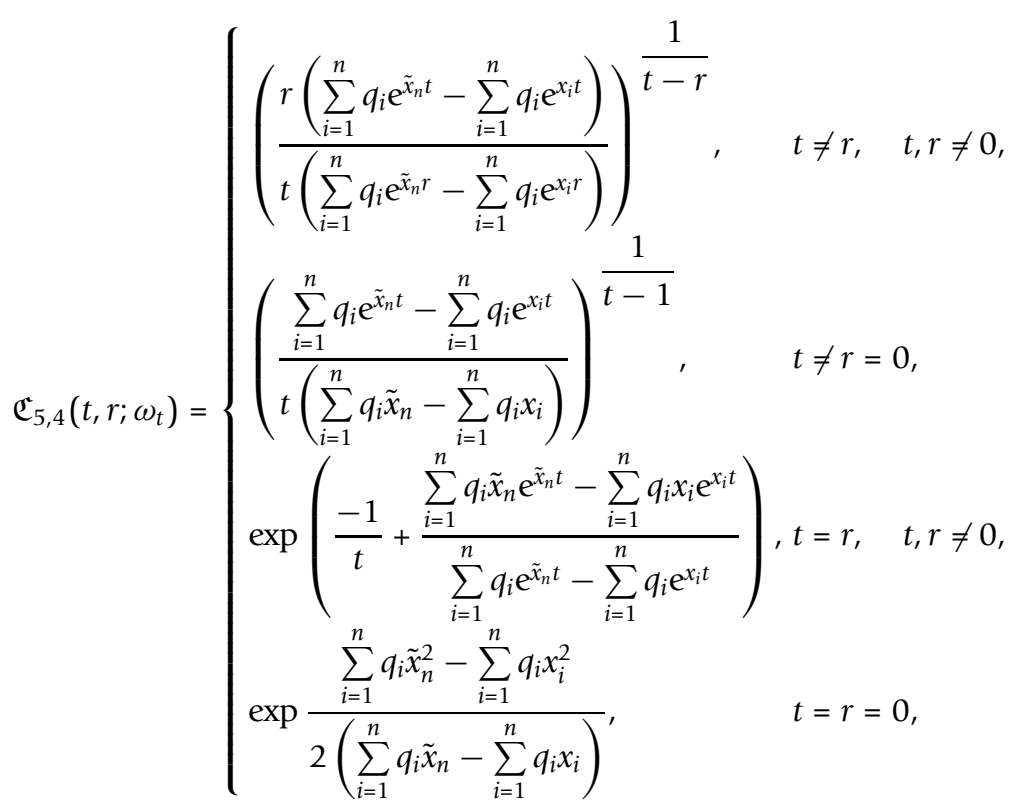

Where $\tilde{x}_{n}=\sum_{i=1}^{n} p_{i} x_{i}$.

\section{Acknowledgements}

The authors wish to thank the anonymous referees for their very careful reading of the manuscript and fruitful comments and suggestions. This research was partially funded by Higher Education Commission, Pakistan. The research of the first author was supported by the Croatian Ministry of Science, Education and Sports under the Research Grant 117-1170889-0888.

\section{Author details}

${ }^{1}$ Abdus Salam School of Mathematical Sciences, GC University, Lahore, Pakistan 'Faculty of Textile Technology, University of Zagreb, Zagreb, Croatia ${ }^{3}$ Department of Mathematics, University of Sargodha, Sargodha, Pakistan

\section{Authors' contributions}

All authors jointly worked on the results and they read and approved the final manuscript.

\section{Competing interests}

The authors declare that they have no competing interests.

Received: 22 March 2011 Accepted: 20 October 2011 Published: 20 October 2011

\section{References}

1. Bernstein, SN: Sur les fonctions absolument monotones. Acta Math. 52, 1-66 (1929). doi:10.1007/BF02592679

2. Widder, DV: The Laplace Transform. Princeton. 1941 (1946)

3. Mitrinović, DS, Pečarić, J, Fink, AM: Classical and New Inequalities in Analysis. Kluwer, The Netherlands (1993) 
4. Pečarić, J, Proschan, F, Tong, YL: Convex Functions, Partial Orderings and Statistical Applications, vol. 187 of Mathematics in Science and Engineering. Academic Press, Boston (1992)

5. Pečarić, J, Ur Rehman, A: On logarithmic convexity for power sums and related results. J Inequal Appl 2008, 9 (2008). Article ID 389410

6. Pečarić, J, Farid, G, Ur Rehman, A: On refinements of Aczél, Popoviciu, Bellmans inequalities and related results. J Inequal Appl 2010, 17 (2010). Article ID 579567

7. Jakšetić, J, Pečarić, J: Exponential convexity method. (in press)

8. Anwar, M, Jakšetić, J, Pečarić, J, Ur Rehman, A: Exponential convexity, positive semi-definite matrices and fundamental inequalities. J Math Inequal. 4(2), 171-189 (2010)

9. Pečarić, J, Ur Rehman, A: On exponentially convexity for power sums and related results. J Math Inequal. (to appear)

10. Pečarić, J, Ur Rehman, A: On logarithmic convexity for power sums and related results II. J Inequal Appl 2008, 12 (2008). Article ID 305623

doi:10.1186/1029-242X-2011-89

Cite this article as: Butt et al:: Exponential convexity of Petrović and related functional. Journal of Inequalities and Applications 2011 2011:89

\section{Submit your manuscript to a SpringerOpen ${ }^{\circ}$} journal and benefit from:

- Convenient online submission

- Rigorous peer review

- Immediate publication on acceptance

- Open access: articles freely available online

- High visibility within the field

- Retaining the copyright to your article

Submit your next manuscript at $\gg$ springeropen.com 